\title{
A supplement to an EOQ model with imperfect quality items, inspection errors, shortage backordering, and sales return
}

\author{
Jia-Tzer Hsu ${ }^{a}$ and Lie-Fern Hsu ${ }^{\text {** }}$
}

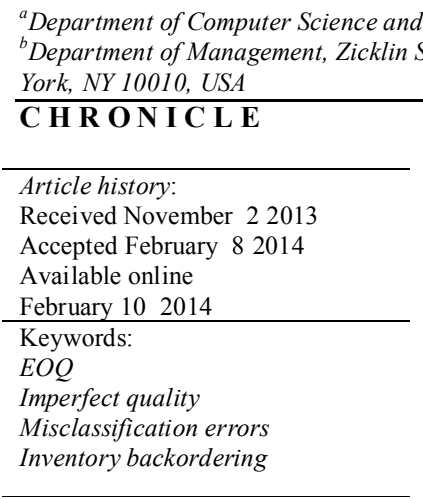

\section{A B S T R A C T}

\begin{abstract}
Hsu and Hsu (2013a) established a closed-form solution for an EOQ model with imperfect quality items, inspection errors, shortage backordering, and sales returns, where the customers who return the defective items will receive full price refunds; i.e., the returned items are not replaced with good items. In this note, we extend Hsu and Hsu's (2013a) work to consider the case that returned items are replaced with good items. A closed-form solution is developed for the optimal order size and the maximum shortage level. Numerical examples are provided to show the differences in the optimal solutions when returned items are replaced, and when they are not.
\end{abstract}

(C) 2014 Growing Science Ltd. All rights reserved

\section{Introduction}

Ever since Harris (1913) presented the famous economic order quantity (EOQ) formula, which is known as the basic EOQ model that appears in every basic textbook covering inventory management, many researchers have extensively studied the economic lot size problems by relaxing some of the assumptions of the basic EOQ model. One of the key assumptions of the basic EOQ model is that the items received are of perfect quality, which is not true in the real industry. Salameh and Jaber (2000) developed an economic order quantity model for the case where a random proportion of the items in a lot are defective. Cárdenas-Barrón (2000) corrected an error in Salameh and Jaber (2000). Goyal and Cárdenas-Barrón (2002) presented a simple approach for determining the economic production quantity of an item with imperfect quality. Papachristos and Konstantaras (2006) discussed the issue of nonshortages in inventory models where the proportion of defective items was a random variable. Eroglu and Ozdemir (2007) extended the model of Salameh and Jaber (2000) by allowing shortages to be fully backordered. Maddah and Jaber (2008) corrected a flaw in the model of Salameh and Jaber (2000) by using the renewal reward theory. Yoo et al. (2009) proposed a profit-maximizing EPQ model that incorporated both imperfect production quality and Type I and Type II inspection errors. Cárdenas-

$*$ Corresponding author

E-mail: Lie-Fern.Hsu@baruch.cuny.edu (L.F. Hsu)

(C) 2014 Growing Science Ltd. All rights reserved.

doi: $10.5267 /$ j.jijiec.2014.2.001 
Barrón (2009) developed an EPQ inventory model with planned backorders for determining the economic production quantity and the size of backorders for a single product, which was made in a single-stage manufacturing process that generated imperfect quality products and required that all defective products be reworked in the same cycle. Liao and Sheu (2011) described an integrated economic production quantity (EPQ) model that incorporated EPQ and maintenance programs. Khan et al. (2011a) reviewed the studies that have extended the EOQ model for imperfect items set forth by Salameh and Jaber (2000).

Khan et al. (2011b) used the models of Salameh and Jaber (2000) and Raouf et al. (1983) to determine an inventory policy for imperfect items subject to Type I and Type II inspection errors. The defective items classified by the inspector and those returned from the market are accumulated and sold at a discounted price at the end of the $100 \%$ screening process. Yassine et al. (2012) extended the basic EPQ with imperfect quality to allow for disaggregation and consolidation of imperfect quality shipments during a single production run and over multiple production runs. Rezari and Salimi (2012) formulated and solved a problem to determine the maximum purchasing price a buyer is willing to pay to a supplier to avoid receiving imperfect items. Yoo et al. (2012) investigated an imperfect quality inventory problem in which imperfect production and inspection processes cause a firm to take actions of internal prevention and external reverse logistics of returns of defective items. They examined and compared the optimal solutions among the basic model and the models with various investment decisions in production and/or inspection processes. Hsu (2012) pointed out a contradiction in Khan et al.'s (2011b) paper; i.e., to obtain the cycle length, the authors assumed that the returned items from the market were replaced with good quality items. However, for the holding cost per cycle, the authors implicitly assumed that the returned items were not replaced with good quality items. Hsu and Hsu (2013a) then extended Khan et al.'s (2011b) model by allowing shortages to be backordered, where they assumed that customers who bought defective items would detect the quality problems and return them to get full price refunds. Chang (2013) corrected some typos that appeared in Yassine et al.'s (2012) consolidating shipments model and presented a heuristic approach to find a good solution of the model. Ouang and Chang (2013) formulated a mathematical model to study the optimal production policy for an EPQ inventory system with imperfect production processes under permissible delay in payments and complete backlogging. Hsu and Hsu (2013b) developed two economic production quantity models with imperfect production processes, inspection errors, planned backorders, and sales returns. In this note, we extend Hsu and Hsu's (2013a) work by assuming that returned items are replaced with good quality items. We develop a closed-form solution for the optimal order size and the maximum shortage level under the situation that returned items are replaced with good ones, and provide numerical examples to show the differences in the optimal solutions of these two models.

\section{Model development}

We use the same assumptions and notation as in Hsu and Hsu (2013a), except for the assumption that instead of giving full price refunds to the customers, the returned items will be replaced with good quality items. The behavior of the inventory level over time is illustrated in Fig.1. By definition, the number of items that are classified as defective include those that are non-defective, $Q(1-p)$, and incorrectly classified as defective (with probability $m_{1}$ ), and those that are defective, $Q p$, and classified as defective (with probability $1-m_{2}$ ); thus, we have

$$
B_{1}=Q(1-p) m_{1}+Q p\left(1-m_{2}\right)
$$

The number of defective items returned from the market are those that are defective, $Q p$, and incorrectly classified as non-defective (with probability $m_{2}$ ); thus

$B_{2}=Q p m_{2}$. 
Since we assume that the defective items returned from the market are replaced with good items, the inventory will be depleted at a rate of $D^{\prime}=D+B_{2} / T$. By definition, the cycle length $T=\left(Q-B_{1}\right) / D^{\prime}$. Substituting $D^{\prime}=D+B_{2} / T$ yields

$T=\frac{\left(Q-B_{1}-B_{2}\right)}{D}=\frac{Q(1-p)\left(1-m_{1}\right)}{D}$.

$D^{\prime}=D+B_{2} / T=D\left(Q-B_{1}\right) /\left(Q-B_{1}-B_{2}\right)=\frac{\left\{(1-p)\left(1-m_{1}\right)+p m_{2}\right\}}{(1-p)\left(1-m_{1}\right)} D=\alpha D$,

with $\alpha=\frac{(1-p)\left(1-m_{1}\right)+p m_{2}}{(1-p)\left(1-m_{1}\right)}$.

\section{Inventory Level}

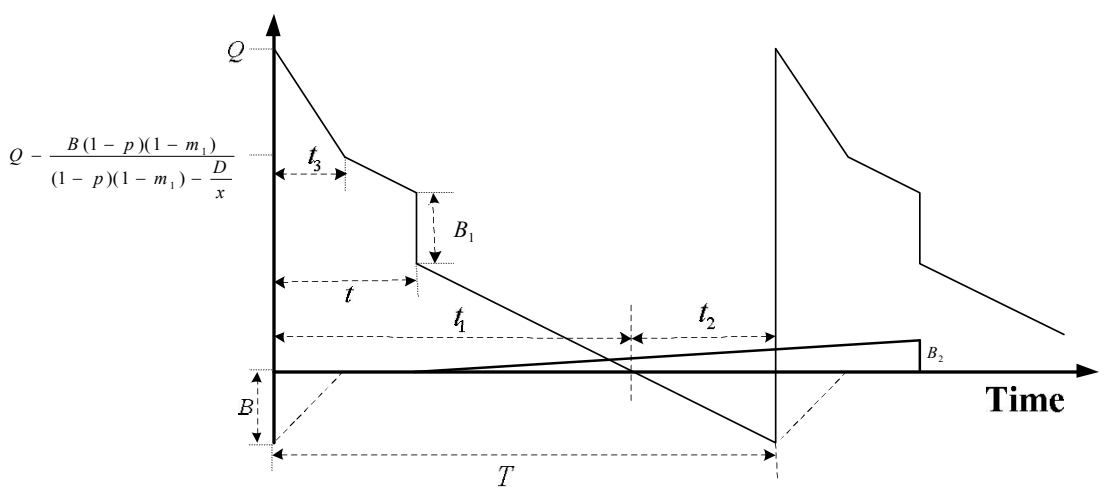

Fig. 1. Behavior of the inventory level over time

Note that in the beginning of each cycle, the inventory level begins with the order quantity $Q$. The $B$ items intended to satisfy the backorders in each cycle will be filled at a rate of $x\left\{1-p-m_{1}+p\left(m_{1}+m_{2}\right\}-\alpha D=x\left\{(1-p)\left(1-m_{1}\right)+p m_{2}\right\}-\alpha D\right.$. Specifically, we have

$$
\begin{aligned}
& t_{1}=\frac{Q\left\{1-p-m_{1}+p\left(m_{1}+m_{2}\right)\right\}-B}{\alpha D}=\frac{Q(1-p)\left(1-m_{1}\right)}{D}-\frac{B(1-p)\left(1-m_{1}\right)}{D\left\{(1-p)\left(1-m_{1}\right)+p m_{2}\right\}}, \\
& t_{2}=\frac{B}{\alpha D}=\frac{B(1-p)\left(1-m_{1}\right)}{D\left\{(1-p)\left(1-m_{1}\right)+p m_{2}\right\}}, \\
& t_{3}=\frac{B}{x\left\{(1-p)\left(1-m_{1}\right)+p m_{2}\right\}-\alpha D}=\frac{B}{\alpha x\left\{(1-p)\left(1-m_{1}\right)-D / x\right\}}, \\
& T=t_{1}+t_{2}=\frac{Q(1-p)\left(1-m_{1}\right)}{D} .
\end{aligned}
$$

After $t_{3}$, the time needed to fill $B$, the maximum shortage level per cycle, the inventory level will be reduced by $B+t_{3} \alpha D=\frac{B(1-p)\left(1-m_{1}\right)}{(1-p)\left(1-m_{1}\right)-\frac{D}{x}}$. The backordering cost per cycle is given as

$\frac{1}{2} b B\left(t_{2}+t_{3}\right)=\frac{1}{2} b B^{2}\left(\frac{(1-p)\left(1-m_{1}\right)}{D\left\{(1-p)\left(1-m_{1}\right)+p m_{2}\right\}}+\frac{(1-p)\left(1-m_{1}\right)}{\left\{(1-p)\left(1-m_{1}\right)+p m_{2}\right\}\left\{x(1-p)\left(1-m_{1}\right)-D\right\}}\right)$

and the holding cost per cycle is 
$h\left\{\left(Q-B_{1}-\frac{1}{2} \frac{B(1-p)\left(1-m_{1}\right)}{\left\{(1-p)\left(1-m_{1}\right)-D / x\right\}}\right) t_{3}+\frac{1}{2}\left(Q-B_{1}-\frac{B(1-p)\left(1-m_{1}\right)}{\left\{(1-p)\left(1-m_{1}\right)-D / x\right\}}\right)\left(t_{1}-t_{3}\right)+\frac{B_{1} Q}{x}+\frac{B_{2} T}{2}\right\}$

$=h\left\{\frac{1}{2}\left(Q-B_{1}\right) t_{3}+\frac{1}{2}\left(Q-B_{1}-\frac{B(1-p)\left(1-m_{1}\right)}{(1-p)\left(1-m_{1}\right)-D / x}\right) t_{1}+\frac{B_{1} Q}{x}+\frac{B_{2} T}{2}\right\}$

$=\frac{h}{2}\left\{Q\left\{(1-p)\left(1-m_{1}\right)+p m_{2}\right\}\left(\frac{B}{\alpha x\left\{(1-p)\left(1-m_{1}\right)-D / x\right\}}\right)\right\}$

$+\frac{h}{2}\left\{Q\left\{(1-p)\left(1-m_{1}\right)+p m_{2}\right\}-\left(\frac{B(1-p)\left(1-m_{1}\right)}{(1-p)\left(1-m_{1}\right)-D / x}\right)\right\}\left(\frac{Q(1-p)\left(1-m_{1}\right)}{D}-\frac{B}{\alpha D}\right)$

$+h\left\{\frac{Q^{2}}{x}\left\{p+m_{1}-p\left(m_{1}+m_{2}\right)\right\}+\frac{Q^{2} p m_{2}(1-p)\left(1-m_{1}\right)}{2 D}\right\}$

$=\frac{h}{2}\left\{\frac{Q B(1-p)\left(1-m_{1}\right)}{x\left\{(1-p)\left(1-m_{1}\right)-D / x\right\}}+\frac{Q^{2}}{D}(1-p)^{2}\left(1-m_{1}\right)^{2}+\frac{Q^{2} p m_{2}(1-p)\left(1-m_{1}\right)}{D}\right\}$

$+\frac{h}{2}\left\{-\frac{Q B(1-p)^{2}\left(1-m_{1}\right)^{2}}{D\left\{(1-p)\left(1-m_{1}\right)-D / x\right\}}-\frac{Q B(1-p)\left(1-m_{1}\right)}{D}+\frac{B^{2}(1-p)^{2}\left(1-m_{1}\right)^{2}}{D\left\{(1-p)\left(1-m_{1}\right)-D / x\right\}\left\{(1-p)\left(1-m_{1}\right)+p m_{2}\right\}}\right\}$

$+h\left\{\frac{Q^{2}}{x}\left\{p+m_{1}-p\left(m_{1}+m_{2}\right)\right\}+\frac{Q^{2} p m_{2}(1-p)\left(1-m_{1}\right)}{2 D}\right\}$.

The total cost per cycle is

$T C(Q, B)=K+c Q+d Q+c_{r} Q(1-p) m_{1}+c_{a} Q p m_{2}$

$+\frac{1}{2} b B^{2}\left(\frac{(1-p)\left(1-m_{1}\right)}{D\left\{1-p-m_{1}+p\left(m_{1}+m_{2}\right)\right\}}+\frac{(1-p)\left(1-m_{1}\right)}{\left\{(1-p)\left(1-m_{1}\right)+p m_{2}\right\}\left\{x(1-p)\left(1-m_{1}\right)-D\right\}}\right)$

$+\frac{h}{2}\left\{\frac{Q B(1-p)\left(1-m_{1}\right)}{x\left\{(1-p)\left(1-m_{1}\right)-D / x\right\}}+\frac{Q^{2}}{D}(1-p)^{2}\left(1-m_{1}\right)^{2}\right\}$

$+\frac{h}{2}\left\{-\frac{Q B(1-p)^{2}\left(1-m_{1}\right)^{2}}{D\left\{(1-p)\left(1-m_{1}\right)-D / x\right\}}-\frac{Q B(1-p)\left(1-m_{1}\right)}{D}+\frac{B^{2}(1-p)^{2}\left(1-m_{1}\right)^{2}}{D\left\{(1-p)\left(1-m_{1}\right)-D / x\right\}\left\{(1-p)\left(1-m_{1}\right)+p m_{2}\right\}}\right\}$

$+h\left\{\frac{Q^{2}}{x}\left\{p+m_{1}-p\left(m_{1}+m_{2}\right)\right\}+\frac{Q^{2} p m_{2}(1-p)\left(1-m_{1}\right)}{D}\right\}$.

Note that the defective items returned from the market will first be sold at the selling price of $s$ per unit; however, when the items are returned from the market they will be replaced with good items and the returned items will be sold at the price of $v$ per unit. Therefore, the total profit per cycle is

$T P(Q, B)=T R(Q, B)-T C(Q, B)=s Q(1-p)\left(1-m_{1}\right)+v Q(1-p) m_{1}+v Q p$

$-K-c Q-d Q-c_{r} Q(1-p) m_{1}-c_{a} Q p m_{2}$

$-\frac{1}{2} b B^{2}\left(\frac{(1-p)\left(1-m_{1}\right)}{D\left\{1-p-m_{1}+p\left(m_{1}+m_{2}\right)\right\}}+\frac{(1-p)\left(1-m_{1}\right)}{\left\{(1-p)\left(1-m_{1}\right)+p m_{2}\right\}\left\{x(1-p)\left(1-m_{1}\right)-D\right\}}\right)$

$-\frac{h}{2}\left\{\frac{Q B(1-p)\left(1-m_{1}\right)}{x\left\{(1-p)\left(1-m_{1}\right)-D / x\right\}}+\frac{Q^{2}}{D}(1-p)^{2}\left(1-m_{1}\right)^{2}\right\}$ 
$-\frac{h}{2}\left\{-\frac{Q B(1-p)^{2}\left(1-m_{1}\right)^{2}}{D\left\{(1-p)\left(1-m_{1}\right)-D / x\right\}}-\frac{Q B(1-p)\left(1-m_{1}\right)}{D}+\frac{B^{2}(1-p)^{2}\left(1-m_{1}\right)^{2}}{D\left\{(1-p)\left(1-m_{1}\right)-D / x\right\}\left\{(1-p)\left(1-m_{1}\right)+p m_{2}\right\}}\right\}$

$-h\left\{\frac{Q^{2}}{x}\left\{p+m_{1}-p\left(m_{1}+m_{2}\right)\right\}+\frac{Q^{2} p m_{2}(1-p)\left(1-m_{1}\right)}{D}\right\}$.

Let $A_{1}=\frac{1}{\alpha}=\frac{(1-p)\left(1-m_{1}\right)}{(1-p)\left(1-m_{1}\right)+p m_{2}}, A_{2}=\frac{(1-p)\left(1-m_{1}\right)}{\left\{(1-p)\left(1-m_{1}\right)+p m_{2}\right\}\left\{x(1-p)\left(1-m_{1}\right)-D\right\}}$,

$A_{3}=\frac{(1-p)\left(1-m_{1}\right)}{(1-p)\left(1-m_{1}\right)-D / x}, A_{4}=\frac{(1-p)^{2}\left(1-m_{1}\right)^{2}}{(1-p)\left(1-m_{1}\right)-D / x}$, and

$A_{5}=\frac{(1-p)^{2}\left(1-m_{1}\right)^{2}}{\left\{(1-p)\left(1-m_{1}\right)-D / x\right\}\left\{(1-p)\left(1-m_{1}\right)+p m_{2}\right\}}$, then the expected profit per cycle is given by

$E[T P(Q, B)]=E[T R(Q, B)]-E[T C(Q, B)]=s Q(1-E[p])\left(1-E\left[m_{1}\right]\right)+v Q(1-E[p]) E\left[m_{1}\right]$

$+v Q E[p]-K-c Q-d Q-c_{r} Q(1-E[p]) E\left[m_{1}\right]-c_{a} Q E[p] E\left[m_{2}\right]$

$-\frac{1}{2} b B^{2}\left(\frac{A_{1}}{D}+A_{2}\right)-\frac{h}{2}\left\{\frac{Q B E\left[A_{3}\right]}{x}+\frac{Q^{2}}{D} E\left[(1-p)^{2}\right] E\left[\left(1-m_{1}\right)^{2}\right]\right\}$

$-\frac{h}{2}\left\{-\frac{Q B E\left[A_{4}\right]}{D}-\frac{Q B(1-E[p])\left(1-E\left[m_{1}\right]\right)}{D}+\frac{B^{2} E\left[A_{5}\right]}{D}\right\}$

$-h\left\{\frac{Q^{2}}{x}\left\{E[p]+E\left[m_{1}\right]-E[p]\left(E\left[m_{1}\right]+E\left[m_{2}\right]\right)\right\}+\frac{Q^{2}\left(E[p]-E\left[p^{2}\right]\right)\left(1-E\left[m_{1}\right]\right) E\left[m_{2}\right]}{D}\right\}$.

From Eq.(3), the expected cycle length is

$$
E[T]=\frac{Q(1-E[p])\left(1-E\left[m_{1}\right]\right)}{D} .
$$

Using the renewal reward theorem, the expected annual profit is

$$
\begin{aligned}
& \operatorname{ETPU}(Q, B)=\frac{E[T P(Q, B)]}{E[T]}=s D+\frac{v D E\left[m_{1}\right]}{\left(1-E\left[m_{1}\right]\right)}+\frac{v D E[p]}{(1-E[p])\left(1-E\left[m_{1}\right]\right)} \\
& -\frac{D\left(\frac{K}{Q}+c+d+c_{r}(1-E[p]) E\left[m_{1}\right]+c_{a} E[p] E\left[m_{2}\right]\right)}{(1-E[p])\left(1-E\left[m_{1}\right]\right)} \\
& -\frac{b B^{2}}{2 Q(1-E[p])\left(1-E\left[m_{1}\right]\right)}\left(E\left[A_{1}\right]+E\left[A_{2}\right] D\right)-\frac{h}{2}\left\{\frac{B D E\left[A_{3}\right] / x+Q E\left[(1-p)^{2}\right] E\left[\left(1-m_{1}\right)^{2}\right]}{(1-E[p])\left(1-E\left[m_{1}\right]\right)}\right\} \\
& -\frac{h}{2}\left\{-\frac{B E\left[A_{4}\right]}{\left(1-E[p)\left(1-E\left[m_{1}\right]\right)\right.}-B+\frac{B^{2} E\left[A_{5}\right]}{Q\left(1-E[p)\left(1-E\left[m_{1}\right]\right)\right.}\right\} \\
& -h Q\left\{\frac{D\left\{E[p]+E\left[m_{1}\right]-E[p]\left(E\left[m_{1}\right]+E\left[m_{2}\right]\right)\right\}}{x(1-E[p])\left(1-E\left[m_{1}\right]\right)}+\frac{\left(E[p]-E\left[p^{2}\right]\right)\left(1-E\left[m_{1}\right]\right) E\left[m_{2}\right]}{(1-E[p])\left(1-E\left[m_{1}\right]\right)}\right\} .
\end{aligned}
$$

By taking the first derivative of $\operatorname{E[PU}(Q, B)]$ with respect to $Q$ and $B$, we have

$$
\frac{\partial E T P U(Q, B)}{\partial Q}=\frac{K D}{Q^{2}(1-E[p])\left(1-E\left[m_{1}\right]\right)}
$$




$$
\begin{aligned}
& +\frac{b B^{2}}{2 Q^{2}(1-E[p])\left(1-E\left[m_{1}\right]\right)}\left(E\left[A_{1}\right]+E\left[A_{2}\right] D\right)-\frac{h}{2}\left\{\frac{E\left[(1-p)^{2}\right] E\left[\left(1-m_{1}\right)^{2}\right]}{(1-E[p])\left(1-E\left[m_{1}\right]\right)}\right\} \\
& +\frac{h}{2} \frac{B^{2} E\left[A_{5}\right]}{Q^{2}\left(1-E[p)\left(1-E\left[m_{1}\right]\right)\right.}-h\left\{\frac{D\left\{E[p]+E\left[m_{1}\right]-E[p]\left(E\left[m_{1}\right]+E\left[m_{2}\right]\right)\right\}}{x(1-E[p])\left(1-E\left[m_{1}\right]\right)}+\frac{\left(E[p]-E\left[p^{2}\right]\right)\left(1-E\left[m_{1}\right]\right) E\left[m_{2}\right]}{(1-E[p])\left(1-E\left[m_{1}\right]\right)}\right\}, \\
& \frac{\partial E T P U(Q, B)}{\partial B}=-\frac{b B}{Q(1-E[p])\left(1-E\left[m_{1}\right]\right)}\left(E\left[A_{1}\right]+E\left[A_{2}\right] D\right) \\
& -\frac{h}{2}\left\{\frac{D E\left[A_{3}\right]}{x(1-E[p])\left(1-E\left[m_{1}\right]\right)}-\frac{E\left[A_{4}\right]}{\left(1-E[p)\left(1-E\left[m_{1}\right]\right)\right.}-1+\frac{2 B E\left[A_{5}\right]}{Q\left(1-E[p)\left(1-E\left[m_{1}\right]\right)\right.}\right\} .
\end{aligned}
$$

Taking the second derivative, we have

$$
\begin{aligned}
& \frac{\partial^{2} E T P U(Q, B)}{\partial Q^{2}}=\frac{-2 K D}{Q^{3}(1-E[p])\left(1-E\left[m_{1}\right]\right)}-\frac{b B^{2}}{Q^{3}(1-E[p])\left(1-E\left[m_{1}\right]\right)}\left(E\left[A_{1}\right]+E\left[A_{2}\right] D\right) \\
& -h \frac{B^{2} E\left[A_{5}\right]}{Q^{3}\left(1-E[p)\left(1-E\left[m_{1}\right]\right)\right.}, \\
& \frac{\partial^{2} E T P U(Q, B)}{\partial B^{2}}=-\frac{b}{Q(1-E[p])\left(1-E\left[m_{1}\right]\right)}\left(E\left[A_{1}\right]+E\left[A_{2}\right] D\right)-\frac{h E\left[A_{5}\right]}{Q\left(1-E[p)\left(1-E\left[m_{1}\right]\right)\right.}, \\
& \frac{\partial^{2} E T P U(Q, B)}{\partial Q \partial B}=\frac{b B}{Q^{2}(1-E[p])\left(1-E\left[m_{1}\right]\right)}\left(E\left[A_{1}\right]+E\left[A_{2}\right] D\right)+\frac{h B E\left[A_{5}\right]}{Q^{2}\left(1-E[p)\left(1-E\left[m_{1}\right]\right)\right.},
\end{aligned}
$$

and

$$
\begin{aligned}
& \left(\frac{\partial^{2} E T P U(Q, B)}{\partial Q^{2}}\right)\left(\frac{\partial^{2} E T P U(Q, B)}{\partial B^{2}}\right)-\left(\frac{\partial^{2} E T P U(Q, B)}{\partial Q \partial B}\right)^{2}=\frac{2 K D h E\left[A_{5}\right]}{Q^{4}(1-E[p])^{2}\left(1-E\left[m_{1}\right]\right)^{2}} \\
& +\frac{2 K D b}{Q^{4}(1-E[p])^{2}\left(1-E\left[m_{1}\right]\right)^{2}}\left(E\left[A_{1}\right]+E\left[A_{2}\right] D\right) .
\end{aligned}
$$

Note that if $D / x<(1-p)\left(1-m_{1}\right)$, we have $\frac{\partial^{2} \operatorname{ETPU}(Q, B)}{\partial Q^{2}}<0, \frac{\partial^{2} \operatorname{ETPU}(Q, B)}{\partial B^{2}}<0$, and $\left(\frac{\partial^{2} E T P U(Q, B)}{\partial Q^{2}}\right)\left(\frac{\partial^{2} E T P U(Q, B)}{\partial B^{2}}\right)-\left(\frac{\partial^{2} E T P U(Q, B)}{\partial Q \partial B}\right)^{2}>0$, which implies that there exist unique values of $Q$ and $B$ that maximize Eq. (12). The optimal solution can be obtained by setting Eq.(13) and Eq. (14) to zero, which lead to

$$
\begin{aligned}
& Q^{*}=\sqrt{\frac{2 K D}{h C-R^{2}\left\{h E\left[A_{5}\right]+b\left(E\left[A_{1}\right]+E\left[A_{2}\right] D\right)\right\}}}, \\
& B^{*}=Q^{*} R,
\end{aligned}
$$

with

$$
R=\frac{h\left\{(1-E[p])\left(1-E\left[m_{1}\right]\right)-E\left[A_{3}\right] D / x+E\left[A_{4}\right]\right\}}{2\left\{h E\left[A_{5}\right]+b\left(E\left[A_{1}\right]+E\left[A_{2}\right] D\right)\right\}},
$$

and

$$
\begin{aligned}
& C=E\left[(1-p)^{2}\right] E\left[\left(1-m_{1}\right)^{2}\right]+2 \frac{D}{x}\left\{E[p]+E\left[m_{1}\right]-E[p]\left(E\left[m_{1}\right]+E\left[m_{2}\right]\right)\right\} \\
& +2\left(E[p]-E\left[p^{2}\right]\right)\left(1-E\left[m_{1}\right]\right) E\left[m_{2}\right] .
\end{aligned}
$$

For the derivations of the expected values of $A_{1}, A_{2}, A_{3}, A_{4}$, and $A_{5}$, please see the Appendix. 


\section{Numerical examples and sensitivity analysis}

We use the same parameters as in Hsu and Hsu (2013a):

Demand rate, $D$

Inspection rate, $x$

Ordering cost, $K$

Holding cost, $h$

Backordering cost, $b$

Inspection cost, $d$

Variable cost, $c$

Selling price of a non-defective item, $s$

Selling price of a defective item, $v$

The cost of accepting a defective item, $c_{a}$

The cost of rejecting a non-defective item, $c_{r}$
$=50,000$ units/year

$=175,200$ units/year

$=\$ 100 /$ order

$=\$ 5 /$ unit $/$ year

$=\$ 10 /$ unit/year

$=\$ 0.5 /$ unit

$=\$ 25 /$ unit

$=\$ 50 /$ unit

$=\$ 20 /$ unit

$=\$ 500 /$ unit

$=\$ 100 /$ unit

If the defective percentage and inspections errors follow a uniform distribution with
$f(p)=\left\{\begin{array}{l}\frac{1}{\beta}, 0 \leq p \leq \beta \\ 0, \text { otherwise }\end{array}\right.$
$f\left(m_{1}\right)=\left\{\begin{array}{c}\frac{1}{\lambda}, 0 \leq m_{1} \leq \lambda \\ 0, \text { otherwise }\end{array}\right.$
$f\left(m_{2}\right)=\left\{\begin{array}{c}\frac{1}{\eta}, 0 \leq m_{2} \leq \eta \\ 0, \text { otherwise }\end{array}\right.$

then we have

$$
\begin{aligned}
& E[p]=\int_{0}^{\beta} p f(p) d p=\int_{0}^{\beta} \frac{p}{\beta} d p=\frac{\beta}{2}, \\
& E\left[p^{2}\right]=\int_{0}^{\beta} p^{2} f(p) d p=\int_{0}^{\beta} \frac{p^{2}}{\beta} d p=\frac{\beta^{2}}{3}, \\
& E\left[(1-p)^{2}\right]=1-\beta+\frac{\beta^{2}}{3}, \\
& E\left[m_{1}\right]=\frac{\lambda}{2}, E\left[\left(1-m_{1}\right)^{2}\right]=1-\lambda+\frac{\lambda^{2}}{3}, \text { and } E\left[m_{2}\right]=\frac{\eta}{2} .
\end{aligned}
$$

Specifically, if $\beta=\lambda=\eta=0.04$, we have $E\left[A_{1}\right]=0.999581, E\left[A_{2}\right]=1.481661, E\left[A_{3}\right]=1.423028$, $E\left[A_{4}\right]=1.3665158, E\left[A_{5}\right]=1.4224298$, and we obtain the expected annual profit as a function of $Q$ and $B$ given in Fig. 2.

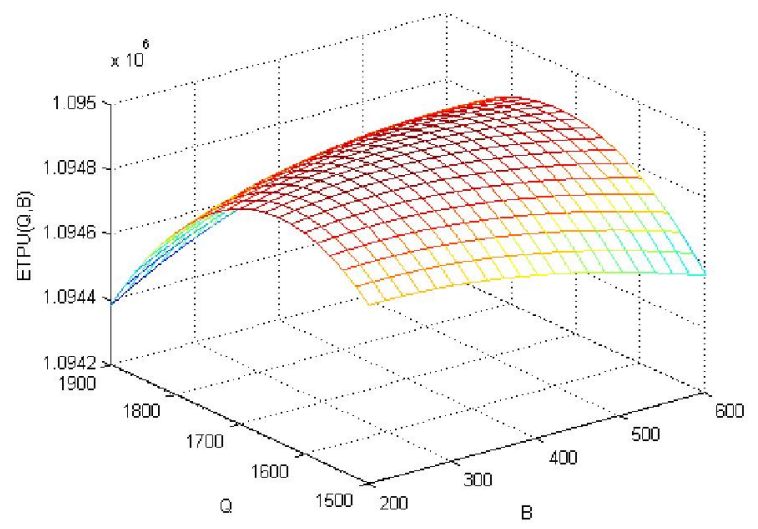

Fig. 2. The expected annual profit 
The three dimensional graph shows that the expected annual profit is concave, and there exist unique solutions of $Q$ and $B$ that maximize the expected annual profit. The optimal solution for the given parameter set is $Q^{*}=1656, B^{*}=373$, and the expected annual profit is $\$ 1,094,918.86$. Note that if the returned items from the market are not replaced with good items, but the customers get full price refunds, then the optimal solution is $Q^{*}=1656, B^{*}=373$, and the expected annual profit is $\$ 1,094,461.93$. Thus, the decision whether or not to replace the returned items with good quality items will not change the decision on the order size and the maximum shortage level for the given parameter set, and the only difference will be in the company's expected annual profit, which will be increased by $0.0417 \%$ if the returned items are replaced with good quality items.

Tables 1 - 5 show the numerical examples for different parameters. It is interesting to note that when the probability of a Type I error is large (e.g., $\lambda$ is greater than or equal to 0.04 ), the expected annual profit becomes negative. This is because in additional to the $\$ 30(s-v)$ per unit loss of incorrectly selling a good item at a defective selling price, there is a penalty cost of $c_{r}=\$ 100$ per unit of incorrectly classifying a non-defective item as defective. From Tables $1-5$ one can see that for all the examples, the expected annual profits (or losses) are slightly larger when the returned items are replaced with good items than when the customers receive a full price refund. The percentage increase in the company's expected annual profit (or loss) is insignificant. For example, it ranges from $0.0207 \%$ to $0.682 \%$ in Table $1 ; 0.0413 \%$ to $0.0553 \%$ in Table $2 ; 0.0209 \%$ to $0.5221 \%$ in Table $3 ; 0.04170 \%$ to $0.04175 \%$ in Table 4 ; and $0.04173 \%$ to $0.041782 \%$ in Table 5 .

\section{Table 1}

Optimal solutions when the defective probability $p$ is uniformly distributed between 0 and $\beta . D=50,000, x$ $=175,200, K=100, h=5, b=10, d=0.5, c=25, s=50, v=20, c_{a}=500, c_{r}=100, \lambda=0.04 ; \eta=0.04$.

\begin{tabular}{|c|c|c|c|c|c|c|c|}
\hline \multirow[b]{2}{*}{$\beta$} & \multicolumn{3}{|c|}{ Without replacement } & \multicolumn{4}{|c|}{ With replacement } \\
\hline & $Q^{*}$ & $B^{*}$ & $\operatorname{ETPU}\left(Q^{*}, B^{*}\right)$ & $Q^{*}$ & $B^{*}$ & $\operatorname{ETPU}\left(Q^{*}, B^{*}\right)$ & $\begin{array}{c}\text { Percentage } \\
\text { increase of } \\
\operatorname{ETPU}\left(Q^{*}, B^{*}\right)\end{array}$ \\
\hline 0.02 & $1,646.92$ & 376.02 & $1,102,873.10$ & $1,647.06$ & 376.02 & $1,103,101.00$ & $0.0207 \%$ \\
\hline 0.04 & $1,655.36$ & 372.62 & $1,094,461.93$ & $1,655.65$ & 372.62 & $1,094,918.86$ & $0.0417 \%$ \\
\hline 0.06 & $1,663.72$ & 369.14 & $1,085,880.33$ & $1,664.15$ & 369.14 & $1,086,567.36$ & $0.0633 \%$ \\
\hline 0.08 & $1,671.98$ & 365.57 & $1,077,123.07$ & $1,672.56$ & 365.56 & $1,078,041.18$ & $0.0852 \%$ \\
\hline 0.10 & $1,680.15$ & 361.90 & $1,068,184.70$ & $1,680.87$ & 361.88 & $1,069,334.80$ & $0.108 \%$ \\
\hline 0.20 & $1,719.20$ & 342.05 & $1,020,569.38$ & $1,720.63$ & 341.97 & $1,022,889.08$ & $0.227 \%$ \\
\hline 0.30 & $1,754.55$ & 319.44 & $967,464.75$ & $1,756.64$ & 319.20 & $970,957.14$ & $0.361 \%$ \\
\hline 0.40 & $1,785.10$ & 293.59 & $907,865.56$ & $1,787.73$ & 293.06 & $912,508.07$ & $0.511 \%$ \\
\hline 0.50 & $1,809.60$ & 263.74 & $840,505.31$ & $1,812.62$ & 262.71 & $846,235.54$ & $0.682 \%$ \\
\hline
\end{tabular}

Table 2

Optimal solutions when the probability of Type I error is uniformly distributed between 0 and $\lambda$. $D=50,000, x$ $=175,200, K=100, h=5, b=10, d=0.5, c=25, s=50, v=20, c_{a}=500, c_{r}=100, \beta=0.04 ; \eta=0.04$

\begin{tabular}{|c|c|c|c|c|c|c|c|}
\hline \multirow[b]{2}{*}{$\lambda$} & \multicolumn{3}{|c|}{ Without replacement } & \multicolumn{3}{|c|}{ With replacement } & \multirow[b]{2}{*}{$\begin{array}{c}\text { Percentage } \\
\text { increase of } \\
\operatorname{ETPU}\left(Q^{*}, B^{*}\right)\end{array}$} \\
\hline & $Q^{*}$ & $B^{*}$ & $\operatorname{ETPU}\left(Q^{*}, B^{*}\right)$ & $Q^{*}$ & $B^{*}$ & $\operatorname{ETPU}\left(Q^{*}, B^{*}\right)$ & \\
\hline 0.02 & $1,646.53$ & 376.04 & $1,149,007.43$ & $1,646.81$ & 376.04 & $1,149,482.24$ & $0.0413 \%$ \\
\hline 0.04 & $1,655.36$ & 372.62 & $1,094,461.93$ & $1,655.65$ & 372.62 & $1,094,918.86$ & $0.0417 \%$ \\
\hline 0.06 & $1,664.11$ & 369.11 & $1,038,791.56$ & $1,664.40$ & 369.11 & $1,039,229.78$ & $0.0422 \%$ \\
\hline 0.08 & $1,672.76$ & 365.51 & $981,961.17$ & $1,673.05$ & 365.51 & $982,379.78$ & $0.0426 \%$ \\
\hline 0.10 & $1,681.31$ & 361.80 & $923,934.12$ & $1,681.60$ & 361.80 & $924,332.20$ & $0.0431 \%$ \\
\hline 0.20 & $1,722.17$ & 341.68 & $614,453.06$ & $1,722.47$ & 341.67 & $614,732.86$ & $0.0455 \%$ \\
\hline 0.30 & $1,759.10$ & 318.62 & $268,556.84$ & $1,759.40$ & 318.59 & $268,686.96$ & $0.0485 \%$ \\
\hline 0.40 & $1,790.85$ & 292.08 & $-120,581.26$ & $1,791.14$ & 292.04 & $-120,641.61$ & $(0.0500 \%)$ \\
\hline 0.50 & $1,816.05$ & 261.25 & $-561,601.91$ & $1,816.33$ & 261.18 & $-561,912.37$ & $(0.0553 \%)$ \\
\hline
\end{tabular}


In terms of the two decision variables (i.e., the order size and the maximum shortage level), the optimal solutions are almost the same whether the customers who return the defective items get full price refunds or good quality items in return. The optimal solutions will be different only if the defective probability $p$ or the probability of a Type II inspection error $m_{2}$ is large. This can be explained intuitively, since the number of items returned from the market is a function of both $p$ and $m_{2}$. From Tables 1 and 3, one can see that there are only slight differences in the optimal order size for the two cases when the defective probability or the probability of Type II inspection error is large. In both cases, the maximum shortage level remains the same.

\section{Table 3}

Optimal solutions when the probability of Type II error is uniformly distributed between 0 and $\eta$. $D=50,000, x$ $=175,200, K=100, h=5, b=10, d=0.5, c=25, s=50, v=20, c_{a}=500, c_{r}=100, \beta=0.04, \lambda=0.04$

\begin{tabular}{|c|c|c|c|c|c|c|c|}
\hline \multirow[b]{2}{*}{$\eta$} & \multicolumn{3}{|c|}{ Without replacement } & \multicolumn{3}{|c|}{ With replacement } & \multirow[b]{2}{*}{$\begin{array}{c}\text { Percentage } \\
\text { increase of } \\
\operatorname{ETPU}\left(Q^{*}, B^{*}\right.\end{array}$} \\
\hline & $Q^{*}$ & $B^{*}$ & $\operatorname{ETPU}\left(Q^{*}, B^{*}\right)$ & $Q^{*}$ & $B^{*}$ & $\operatorname{ETPU}\left(Q^{*}, B^{*}\right)$ & \\
\hline 0.02 & $1,655.76$ & 372.60 & $1,099,896.39$ & $1,655.90$ & 372.60 & $1,100,125.99$ & $0.0209 \%$ \\
\hline 0.04 & $1,655.36$ & 372.62 & $1,094,461.93$ & $1,655.65$ & 372.62 & $1,094,918.86$ & $0.0417 \%$ \\
\hline 0.06 & $1,654.97$ & 372.64 & $1,089,029.74$ & $1,655.40$ & 372.64 & $1,089,711.74$ & $0.0626 \%$ \\
\hline 0.08 & $1,654.57$ & 372.67 & $1,083,599.81$ & $1,655.15$ & 372.67 & $1,084,504.62$ & $0.0835 \%$ \\
\hline 0.10 & $1,654.17$ & 372.69 & $1,078,172.13$ & $1,654.90$ & 372.69 & $1,079,297.50$ & $0.1044 \%$ \\
\hline 0.20 & $1,652.19$ & 372.80 & $1,051,067.58$ & $1,653.64$ & 372.79 & $1,053,261.90$ & $0.2088 \%$ \\
\hline 0.30 & $1,650.21$ & 372.90 & $1,024,019.28$ & $1,652.38$ & 372.90 & $1,027,226.30$ & $0.3132 \%$ \\
\hline 0.40 & $1,648.23$ & 373.00 & $997,027.05$ & $1,651.13$ & 373.01 & $1,001,190.70$ & $0.4176 \%$ \\
\hline 0.50 & $1,646.25$ & 373.11 & $970,090.72$ & $1,649.88$ & 373.11 & $975,155.11$ & $0.5221 \%$ \\
\hline
\end{tabular}

\section{Table 4}

Optimal solutions for different holding cost, $D=50,000, x=175,200, K=100, b=10, d=0.5, c=25, s=50, v=20, c_{a}=$ $500, c_{r}=100, \beta=0.04, \lambda=0.04, \eta=0.04$

\begin{tabular}{|c|c|c|c|c|c|c|c|}
\hline \multirow[b]{2}{*}{$h$} & \multicolumn{3}{|c|}{ Without replacement } & \multicolumn{4}{|c|}{ With replacement } \\
\hline & $Q^{*}$ & $B^{*}$ & $\operatorname{ETPU}\left(Q^{*}, B^{*}\right)$ & $Q^{*}$ & $B^{*}$ & $\operatorname{ETPU}\left(Q^{*}, B^{*}\right)$ & $\begin{array}{c}\text { Percentage } \\
\text { increase of } \\
\operatorname{ETPU}\left(Q^{*}, B^{*}\right)\end{array}$ \\
\hline 1 & $3,357.37$ & 206.11 & $1,097,649.33$ & $3,358.02$ & 206.12 & $1,098,107.10$ & $0.04170 \%$ \\
\hline 2 & $2,442.65$ & 274.92 & $1,096,488.43$ & $2,443.12$ & 274.92 & $1,096,945.92$ & $0.04172 \%$ \\
\hline 3 & $2,045.83$ & 318.82 & $1,095,661.96$ & $2,046.21$ & 318.82 & $1,096,119.23$ & $0.04173 \%$ \\
\hline 4 & $1,812.78$ & 349.76 & $1,095,007.92$ & $1,813.10$ & 349.76 & $1,095,465.01$ & $0.04174 \%$ \\
\hline 5 & $1,655.36$ & 372.62 & $1,094,461.93$ & $1,655.65$ & 372.62 & $1,094,918.86$ & $0.04175 \%$ \\
\hline
\end{tabular}

\section{Table 5}

Optimal solutions for different backordering cost, $D=50,000, x=175,200, K=100, h=5, d=0.5, c=$ $25, s=50, v=20, c_{a}=500, c_{r}=100, \beta=0.04, \lambda=0.04, \eta=0.04$

\begin{tabular}{|c|c|c|c|c|c|c|c|}
\hline \multirow[b]{2}{*}{$b$} & \multicolumn{3}{|c|}{ Without replacement } & \multicolumn{3}{|c|}{ With replacement } & \multirow[b]{2}{*}{$\begin{array}{c}\text { Percentage } \\
\text { increase of } \\
\operatorname{ETPU}\left(Q^{*}, B^{*}\right.\end{array}$} \\
\hline & $Q^{*}$ & $B^{*}$ & $\operatorname{ETPU}\left(Q^{*}, B^{*}\right)$ & $Q^{*}$ & $B^{*}$ & $\operatorname{ETPU}\left(Q^{*}, B^{*}\right)$ & \\
\hline 5 & $1,793.55$ & 605.59 & $1,094,946.38$ & $1,793.83$ & 605.58 & $1,095,403.30$ & $0.041730 \%$ \\
\hline 10 & $1,655.36$ & 372.62 & $1,094,461.93$ & $1,655.65$ & 372.62 & $1,094,918.86$ & $0.041749 \%$ \\
\hline 15 & $1,597.26$ & 269.66 & $1,094,233.20$ & $1,597.55$ & 269.66 & $1,094,690.14$ & $0.041759 \%$ \\
\hline 20 & $1,565.20$ & 211.40 & $1,094,099.74$ & $1,565.49$ & 211.40 & $1,094,556.67$ & $0.041763 \%$ \\
\hline$\infty$ & $1,453.91$ & 0 & $1,093,590.73$ & $1,454.20$ & 0 & $1,094,047.65$ & $0.041782 \%$ \\
\hline
\end{tabular}

In the real world, we know that some of the customers who return a defective item will ask for a full price refund, while others are willing to exchange their defective item for a good one. The numerical examples show that there is no significant difference in terms of the optimal order size and the 
maximum shortage level for the two cases. Thus, no matter what the percentages of customers who return the items and ask for a refund or an exchange are, the management can use one of the two models to decide the order size and the maximum shortage level as long as the defective probability and the probability of a Type II inspection error are small.

\section{Summary and conclusion}

In this note, we extend Hsu and Hsu's (2013a) work by assuming that returned items are replaced with good quality items. We develop a closed-form solution for the optimal order size and the maximum shortage level under the situation that returned items are replaced with good ones. Numerical examples show that although the expected annual profit is always slightly higher for the case when customers who return items receive replacement items rather than full price refunds, the solutions are almost the same for the two cases in terms of the optimal order size and the maximum shortage level.

In the real world, some customers who bought defective items will ask for a full price refund, while others are willing to exchange their defective item for a good item. It may be difficult to know in advance if a customer who returns the item will ask for a full price refund or an exchange. Since our numerical analysis shows that there is no significant difference in terms of the optimal order size and the maximum shortage level, the management can just use one of the two models to make a decision.

\section{Appendix: Gaussian Quadrature in two dimensions (Hughes 1987)}

Gaussian rules for integrals in several dimensions are constructed by employing one-dimensional Gaussian rules on each coordinate separately. For example, in two dimensions:

$$
\int_{-1-1}^{1} \int_{1}^{1} g(\xi, \phi) d \xi d \phi \cong \sum_{i=1}^{n_{\text {int }}^{(1)} \sum_{j=1}^{(2)}} g\left(\widetilde{\xi}_{i}^{(1)}, \widetilde{\phi}_{j}^{(2)}\right) W_{i}^{(1)} W_{j}^{(2)}
$$

where $n_{\text {int }}^{(1)}$ and $n_{\text {int }}^{(2)}$ are the numbers of integration points, $\widetilde{\xi}_{i}^{(1)}$ and $\widetilde{\phi}_{j}^{(2)}$ are the coordinates of integration points, and $W_{i}^{(1)}$ and $W_{j}^{(2)}$ are the weights of integration points in $\xi$-coordinate and $\phi$ coordinate, respectively. In terms of a single index, we would have

$$
\int_{-1-1}^{1} \int_{-1}^{1} g(\xi, \phi) d \xi d \phi \cong \sum_{i=1}^{n_{\text {int }}} g\left(\widetilde{\xi}_{i}, \widetilde{\phi}_{i}\right) W_{i}
$$

where $n_{\text {int }}=n_{\text {int }}^{(1)} n_{\text {int }}^{(2)}, \widetilde{\xi}_{i}=\widetilde{\xi}_{i}^{(1)}, \widetilde{\phi}_{i}=\widetilde{\phi}_{j}^{(2)}, W_{i}=W_{i}^{(1)} W_{j}^{(2)}$.

In particular, for two-point rule $\left(n_{\text {int }}=2 \cdot 2=4\right)$,

$$
\begin{aligned}
& \widetilde{\xi}_{1}=\frac{-1}{\sqrt{3}}, \widetilde{\xi}_{2}=\frac{1}{\sqrt{3}}, \widetilde{\xi}_{3}=\frac{1}{\sqrt{3}}, \widetilde{\xi}_{4}=\frac{-1}{\sqrt{3}}, \\
& \widetilde{\phi}_{1}=\frac{-1}{\sqrt{3}}, \widetilde{\phi}_{2}=\frac{-1}{\sqrt{3}}, \widetilde{\phi}_{3}=\frac{1}{\sqrt{3}}, \widetilde{\phi}_{4}=\frac{1}{\sqrt{3}}, \\
& W_{1}=W_{2}=W_{3}=W_{4}=1, \\
& \int_{-1-1}^{1} \int_{1}^{1} g(\xi, \phi) d \xi d \phi=g\left(\frac{-1}{\sqrt{3}}, \frac{-1}{\sqrt{3}}\right)+g\left(\frac{1}{\sqrt{3}}, \frac{-1}{\sqrt{3}}\right)+g\left(\frac{1}{\sqrt{3}}, \frac{1}{\sqrt{3}}\right)+g\left(\frac{-1}{\sqrt{3}}, \frac{1}{\sqrt{3}}\right) .
\end{aligned}
$$

For $A_{1}=\frac{(1-p)\left(1-m_{1}\right)}{(1-p)\left(1-m_{1}\right)+p m_{2}}$, the expected value of $A_{1}$ is

$$
E\left[A_{1}\right]=\frac{1}{\beta \lambda \eta} \int_{0}^{\lambda \beta} \int_{0}^{\beta \eta} \frac{(1-p)\left(1-m_{1}\right)}{(1-p)\left(1-m_{1}\right)+p m_{2}} d m_{2} d p d m_{1}=\frac{1}{\beta \lambda \eta} \int_{0}^{\lambda \beta} \int_{0}^{\beta} \frac{(1-p)\left(1-m_{1}\right)}{p} \cdot \ln \left(\frac{\eta p}{(1-p)\left(1-m_{1}\right)}+1\right) d p d m_{1} \text {. }
$$


To change the integration range $p:[0, \beta] \rightarrow \xi:[-1,1]$ and $m_{1}:[0, \lambda] \rightarrow \phi:[-1,1]$, we need to let $p=\frac{\beta}{2}(1+\xi)$, $m_{1}=\frac{\lambda}{2}(1+\phi)$ and $d p d m_{1}=J \cdot d \xi d \phi$, where the Jacobian determinant $J=\operatorname{det}\left[\begin{array}{cc}p_{, \xi} & p_{, \phi} \\ m_{1, \xi} & m_{1, \phi}\end{array}\right]=\frac{\beta \lambda}{4}$. Thus, Eq.(A4) becomes

$$
\begin{aligned}
E\left[A_{1}\right] & =\frac{1}{\beta \lambda \eta} \int_{-1}^{1} \int_{-1}^{1} \frac{\left[1-\frac{\beta}{2}(1+\xi)\right]\left[1-\frac{\lambda}{2}(1+\phi)\right]}{\frac{\beta}{2}(1+\xi)} \cdot \ln \left(\frac{\frac{\beta \eta}{2}(1+\xi)}{\left(1-\frac{\beta}{2}(1+\xi)\right)\left(1-\frac{\lambda}{2}(1+\phi)\right)}+1\right) \cdot \frac{\beta \lambda}{4} d \xi d \phi \\
& =\frac{1}{2 \beta \eta} \int_{-1}^{1} \int_{-1}^{1} \frac{\left[1-\frac{\beta}{2}(1+\xi)\right]\left[1-\frac{\lambda}{2}(1+\phi)\right]}{(1+\xi)} \cdot \ln \left(\frac{\frac{\beta \eta}{2}(1+\xi)}{\left(1-\frac{\beta}{2}(1+\xi)\right)\left(1-\frac{\lambda}{2}(1+\phi)\right)}+1\right) d \xi d \phi .
\end{aligned}
$$

Using the two-point rule $\left(n_{\text {int }}=2 \cdot 2=4\right)$, Eq.(A5) specializes to $E\left[A_{1}\right]=\frac{1}{2 \beta \eta}\left[g_{1}\left(\frac{-1}{\sqrt{3}}, \frac{-1}{\sqrt{3}}\right)+g_{1}\left(\frac{1}{\sqrt{3}}, \frac{-1}{\sqrt{3}}\right)+g_{1}\left(\frac{1}{\sqrt{3}}, \frac{1}{\sqrt{3}}\right)+g_{1}\left(\frac{-1}{\sqrt{3}}, \frac{1}{\sqrt{3}}\right)\right]$,

where

$g_{1}(\xi, \phi)=\frac{\left(1-\frac{\beta}{2}(1+\xi)\right)\left(1-\frac{\lambda}{2}(1+\phi)\right)}{(1+\xi)} \cdot \ln \left(\frac{\frac{\beta \eta}{2}(1+\xi)}{\left(1-\frac{\beta}{2}(1+\xi)\right)\left(1-\frac{\lambda}{2}(1+\phi)\right)}+1\right)$

Similarly, $\quad$ for $A_{2}=\frac{(1-p)\left(1-m_{1}\right)}{\left\{(1-p)\left(1-m_{1}\right)+p m_{2}\right\}\left\{(1-p)\left(1-m_{1}\right)-\frac{D}{x}\right\}}, A_{3}=\frac{(1-p)\left(1-m_{1}\right)}{(1-p)\left(1-m_{1}\right)-\frac{D}{x}}$, $A_{5}=\frac{(1-p)^{2}\left(1-m_{1}\right)^{2}}{\left\{(1-p)\left(1-m_{1}\right)-\frac{D}{x}\right\}\left\{(1-p)\left(1-m_{1}\right)+p m_{2}\right\}}$, the expected values of $A_{2}, A_{3}$, and $A_{5}$ are

$E\left[A_{2}\right]=\frac{1}{2 \beta \eta}\left[g_{2}\left(\frac{-1}{\sqrt{3}}, \frac{-1}{\sqrt{3}}\right)+g_{2}\left(\frac{1}{\sqrt{3}}, \frac{-1}{\sqrt{3}}\right)+g_{2}\left(\frac{1}{\sqrt{3}}, \frac{1}{\sqrt{3}}\right)+g_{2}\left(\frac{-1}{\sqrt{3}}, \frac{1}{\sqrt{3}}\right)\right]$

$E\left[A_{3}\right]=\frac{1}{4}\left[g_{3}\left(\frac{-1}{\sqrt{3}}, \frac{-1}{\sqrt{3}}\right)+g_{3}\left(\frac{1}{\sqrt{3}}, \frac{-1}{\sqrt{3}}\right)+g_{3}\left(\frac{1}{\sqrt{3}}, \frac{1}{\sqrt{3}}\right)+g_{3}\left(\frac{-1}{\sqrt{3}}, \frac{1}{\sqrt{3}}\right)\right]$

$E\left[A_{5}\right]=\frac{1}{2 \beta \eta}\left[g_{5}\left(\frac{-1}{\sqrt{3}}, \frac{-1}{\sqrt{3}}\right)+g_{5}\left(\frac{1}{\sqrt{3}}, \frac{-1}{\sqrt{3}}\right)+g_{5}\left(\frac{1}{\sqrt{3}}, \frac{1}{\sqrt{3}}\right)+g_{5}\left(\frac{-1}{\sqrt{3}}, \frac{1}{\sqrt{3}}\right)\right]$

where

$g_{2}(\xi, \phi)=\frac{\left(1-\frac{\beta}{2}(1+\xi)\right)\left(1-\frac{\lambda}{2}(1+\phi)\right)}{\left\{\left(1-\frac{\beta}{2}(1+\xi)\right)\left(1-\frac{\lambda}{2}(1+\phi)\right)-\frac{D}{x}\right\}(1+\xi)} \cdot \ln \left(\frac{\frac{\beta \eta}{2}(1+\xi)}{\left(1-\frac{\beta}{2}(1+\xi)\right)\left(1-\frac{\lambda}{2}(1+\phi)\right)}+1\right)$

$g_{3}(\xi, \phi)=\frac{\left(1-\frac{\beta}{2}(1+\xi)\right)\left(1-\frac{\lambda}{2}(1+\phi)\right)}{\left(1-\frac{\beta}{2}(1+\xi)\right)\left(1-\frac{\lambda}{2}(1+\phi)\right)-\frac{D}{x}}$,

$g_{5}(\xi, \phi)=\frac{\left(1-\frac{\beta}{2}(1+\xi)\right)^{2}\left(1-\frac{\lambda}{2}(1+\phi)\right)^{2}}{\left\{\left(1-\frac{\beta}{2}(1+\xi)\right)\left(1-\frac{\lambda}{2}(1+\phi)\right)-\frac{D}{x}\right\}(1+\xi)} \cdot \ln \left(\frac{\frac{\beta \eta}{2}(1+\xi)}{\left(1-\frac{\beta}{2}(1+\xi)\right)\left(1-\frac{\lambda}{2}(1+\phi)\right)}+1\right)$.

For $A_{4}=\frac{(1-p)^{2}\left(1-m_{1}\right)^{2}}{(1-p)\left(1-m_{1}\right)-\frac{D}{x}}$, we can obtain

$A_{4}=(1-p)\left(1-m_{1}\right)+\left(\frac{D}{x}\right) \cdot A_{3}$.

Therefore, the expected value of $A_{4}$ can be expressed as

$$
E\left[A_{4}\right]=E\left[(1-p)\left(1-m_{1}\right)\right]+\left(\frac{D}{x}\right) E\left[A_{3}\right]=\left(1-\frac{\beta}{2}\right)\left(1-\frac{\lambda}{2}\right)+\left(\frac{D}{x}\right) E\left[A_{3}\right]
$$




\section{References}

Cárdenas-Barrón, L.E., (2000). Observation on: "Economic production quantity model for items with imperfect quality" [Int. J. Prod. Econ. 64 (2000) 59-64]. International Journal of Production Economics, 67 (2), 201.

Cárdenas-Barrón, L.E., (2009). Economic production quantity with rework process at a single stage manufacturing system with planned backorders. Computers and Industrial Engineering, 57 (3), 11051113.

Chang, H.C., (2013). An economic production quantity model with consolidating shipments of imperfect quality items: A note. International Journal of Production Economics, 144 (2), 507-509.

Eroglu, A., \& Ozdemir, G., (2007). An economic order quantity model with defective items and shortages. International Journal of Production Economics, 106 (2), 544-549.

Goyal, S. K., \& Cárdenas-Barrón, L. E., (2002). Note on: economic production quantity model for items with imperfect quality - a practical approach. International Journal of Production Economics, 77, 8587.

Harris, F.W., (1913). How many parts to make at once. Factory, The Magazine of Management, 10 (2), $135-$ $136, \& 152$.

Hsu, L.F., (2012). A note on "An economic order quantity (EOQ) for items with imperfect quality and inspection errors." International Journal of Industrial Engineering Computations, 3 (4), 695-702.

Hsu, J.T., \& Hsu, L.F., (2013a). An EOQ model with imperfect quality items, inspection errors, shortage backordering, and sales return. International Journal of Production Economics, 143 (1), 162-170.

Hsu, J.T., \& Hsu, L.F., (2013b). Two EPQ models with imperfect production processes, inspection errors, planned backorders, and sales returns. Computers \& Industrial Engineering, 64 (1),389-402.

Hughes, T.J.R., (1987). The Finite Element Method. Prentice-Hall, Inc., Englewood Cliffs, NJ.

Khan, M., Jaber, M.Y., Guiffrida, A.L., \& Zolfaghari, S., (2011a). A review of the extensions of a modified EOQ model for imperfect quality items. International Journal of Production Economics, 132 (1), 1-12.

Khan, M., Jaber, M.Y., \& Bonney, M., (2011b). An economic order quantity (EOQ) for items with imperfect quality and inspection errors. International Journal of Production Economics, 133 (1), 113118.

Liao, G.L., \& Sheu, S.H., (2011). Economic production quantity model for randomly failing production process with minimal repair and imperfect maintenance. International Journal of Production Economics, 130 (1), 118-124.

Maddah, B., \& Jaber, M.Y., (2008). Economic order quantity for items with imperfect quality: revisited. International Journal of Production Economics, 112 (2), 808-815.

Ouang, L.Y., \& Chang, C.T., (2013). Optimal production lot with imperfect production process under permissible delay in payments and complete backlogging. International Journal of Production Economics, 144 (2), 610-617.

Papachristos, S., \& Konstantaras, I., (2006). Economic ordering quantity models for items with imperfect quality. International Journal of Production Economics, 100 (1), 148-154.

Raouf, A., Jain, J.K., \& Sathe, P.T., (1983). A cost-minimization model for multicharacteristic component inspection. IIE Transactions, 15 (3), 187-194.

Rezaie, J., \& Salimi, N., (2012). Economic order quantity and purchasing price for items with imperfect quality when inspection shifts from buyer to supplier. International Journal of Production Economics, 137 (1), 11-18.

Salameh, M.K., \& Jaber, M.Y., (2000). Economic production quantity model for items with imperfect quality. International Journal of Production Economics, 64 (1), 59-64.

Yassine, A., Maddah, B., \& Salameh, M., (2012). Disaggregation and consolidation of imperfect quality shipments in an extended EPQ model. International Journal of Production Economics, 135 (1), 345-352.

Yoo, S.H., Kim, D., \& Park, M.S., (2009). Economic production quantity model with imperfect-quality items, two-way imperfect inspection and sales return. International Journal of Production Economics, $121(1), 255-265$.

Yoo, S.H., Kim, D., \& Park, M.S., (2012). Lot sizing and quality investment with quality cost analyses for imperfect production and inspection processes with commercial return. International Journal of Production Economics, 140 (2), 922-933. 